\title{
Dos cartas neolatinas inéditas entre los humanistas Lorenzo Ramírez de Prado (1583-1658) e Isaac Casaubon (1559-1614). Edición, traducción y comentario
}

\author{
Two Unpublished Neo-Latin Letters between \\ the Humanists Lorenzo Ramírez de Prado (1583-1658) \\ and Isaac Casaubon (1559-1614). \\ Edition, Translation and Commentary
}

\author{
Juan Ramón BALLesteros SÁNCHeZ \\ https://orcid.org/0000-0002-7168-293X \\ Universidad Pablo de Olavide, España \\ jrbalsan@upo.es
}

RESUMEN: El texto ofrece la primera edición, traducción castellana y comentario de dos cartas neolatinas intercambiadas entre Lorenzo Ramírez de Prado (15831658) e Isaac Casaubon (1559-1614). Estas cartas fueron las primeras que se enviaron sus autores. Su estudio ilustra los mecanismos por medio de los cuales se constituyó la República de las Letras en el Humanismo tardío y permite reconstruir la relación entre ambos eruditos.

Palabras clave: Lorenzo Ramírez de Prado, Isaac Casaubon, correspondencia erudita humanística

AвstRAct: The text offers the first edition, Spanish translation and commentary of two Neo-Latin letters exchanged between Lorenzo Ramírez de Prado (15831658) and Isaac Casaubon (1559-1614). These letters were the first their authors sent each other. His study illustrates the mechanisms by which the Republic of Letters is managed in late Humanism and allows the reconstruction of the relationship between both scholars.

Keywords: Lorenzo Ramírez de Prado, Isaac Casaubon, Humanistic Scholarly Correspondence

RECIBIDO: 05/09/2019 • ACEPTADO: 16/10/2019 • VERSIÓN FINAL: 18/12/2019 


\section{INTRODUCCIÓN}

En un artículo aparecido en 1998 en la revista Humanistica Lovaniensia, el profesor José Solís de los Santos publicó una descripción del manuscrito 2598 de la Biblioteca General de la Universidad de Salamanca (= BU ms. 2598). Este documento, titulado "Epistolario Selecto de Diversos Varones", contiene 80 misivas eruditas escritas en latín - sólo la carta $57=79$, con un tratadillo en latín de Francisco Cascales (1567-1642), tiene un encabezamiento en castellano- - Las epístolas fueron coleccionadas por el propietario del códice: Lorenzo Ramírez de Prado (1583-1658). No todas las misivas están datadas, pero las que poseen fecha permiten situar este epistolario en el lustro transcurrido entre septiembre de 1601 (carta 29) y noviembre de 1606 (carta 76). Todas las misivas de BU ms. 2598 son transcripciones. Solís sospechó que la transcripción, hecha por dos manos diferentes - la primera hasta el f. 82 y la segunda a partir del $83-$, fue realizada a la muerte de Ramírez de Prado, "mientras se procedía a la catalogación de la biblioteca y archivo del docto consejero [i. e. Ramírez de Prado], bien por escribientes de la comisión inquisitorial que llevaba a cabo el expurgo, o bien por encargo de su viuda, quien se apresuró a deshacerse de tan enorme cúmulo de libros". ${ }^{1}$

Entre las cartas de BU ms. 2598 existen dos que permiten reconstruir una breve relación epistolar entre Lorenzo Ramírez de Prado e Isaac Casaubon. Ninguna de ellas, según mis conocimientos, ha sido editada hasta ahora. La carta 58, sin fecha, es la primera que Ramírez de Prado envió a Casaubon. Un terminus ante quem para la fecha de la misma puede deducirse de otra carta del ms. salmantino: la 80, redactada desde París por Johannes Bodecker, el 1 de agosto de 1606. Este Johannes Bodecker, según se explica en la epístola 58, fue quien convenció a Ramírez de Prado para que escribiera a Casaubon y fue el correo de la carta 58. Según cuenta en su misiva a Ramírez de Prado, Bodecker también debía encargarse de entregar la carta 59 de Ramírez de Prado a Justo Lipsio - como se verá más adelante ambas compartían una intención similar-, pero la muerte de Lipsio a finales de marzo de 1606 le impidió hacerlo. Como las cartas de Ramírez de Prado a Lipsio y a Casaubon viajaron juntas en el equipaje de Bodecker hay que deducir que la 58 debió escribirse antes del fallecimiento de Lipsio, acaecido el 23 de marzo de 1606. Las epístolas 58 y 59 tenían la intención de presentar a Lipsio y a Casaubon un opúsculo de Ramírez de Prado sobre cierto pasaje de la Historia Natural de Plinio. ${ }^{2}$ Ramírez de Prado recabó apoyos para este trabajo desde agosto de 1605 por medio de otras misivas copiadas en BU ms. 2598. Por esta razón, Solís dató la carta 59 a Lipsio ca. agosto 1605.

\footnotetext{
${ }^{1}$ Cf. Solís de los Santos 1998, p. 310.

${ }^{2}$ Cf. los comentarios a I, 15-16 y a II, 10-11 de esta edición.
} 
El profesor Solís también fue consciente, no obstante, de que en la carta 61 Bartolomé Morlanes (1576-1649) propuso a Ramírez de Prado remitir a Justo Lipsio la polémica sobre el pasaje pliniano y, naturalmente, su trabajo sobre la misma (f. 69r: Sed litem hanc dirimendam relinquire Lipsio: cui alii melius in hoc aevo?). Esta misiva tiene como fecha 5 de noviembre de 1605 y, aunque en la respuesta de Ramírez de Prado a Morlanes (carta 62, sin fecha) no se aluda a las cartas a Lipsio y a Casaubon, en mi opinión, constituye el terminus post quem de ambas epístolas. Las 58 y 59 de BU ms. 2598 debieron escribirse, por lo tanto, entre noviembre de 1605 y marzo de 1606.

La segunda carta es la respuesta de Casaubon a la de Ramírez de Prado. Se trata de la epístola 78 de BU ms. 2598 que está fechada ultro Lutetiae Parisiorum, el 31 de agosto de 1606, por lo tanto, aproximadamente un mes después de que Bodecker redactara la suya informando que Casaubon había recibido la carta de Ramírez de Prado.

Estas dos misivas ofrecen un testimonio - quizá el más insospechadode los relativamente frecuentes contactos interconfesionales existentes en la Europa de los humanistas. ${ }^{3}$ Son las primeras cartas que intercambiaron Ramírez de Prado y Casaubon y presentan la personalidad de ambos humanistas al inicio de su relación. Ramírez de Prado, un joven de 22 años, ambicioso, impulsivo y brillante, ha decidido empezar una carrera en el mundo de las letras clásicas. ${ }^{4}$ Trabaja en un comentario a Marcial del que - a diferencia de lo que ocurre con Lipsio - nada dice a Casaubon. ${ }^{5}$ Se muestra humilde y respetuoso ante el gran erudito francés. Lo adula mediante varias citas tomadas de sus comentarios a Persio. Casaubon, por su parte, lleva media década viviendo en París, donde se ha instalado gracias a la protección del rey Enrique IV. ${ }^{6}$ En plena madurez - Casaubon está trabajando en ese tiempo en su magna obra sobre Polibio - , recibe con entusiasmo la carta de Ramírez de Prado y la posibilidad que ésta le ofrece de ampliar al otro lado de los Pirineos la red de su correspondencia erudita. En su misiva a Ramírez de Prado se muestra sencillo y modesto, pero también ofrece alguno de esos "significados ocultos bajo capas de erudición" que proporcionan a su epistolario un tono característico. ${ }^{7}$

\footnotetext{
${ }^{3}$ Cf. Van Miert 2009.

${ }^{4}$ En la respuesta a la carta de Morlanes, Ramírez resume sus proyectos inmediatos del siguiente modo: Scopus item cui omnia dirigo est Iurisconsultos illustrare, varios auctores interpretari ac corrigere si studia in umbra educata non fuerint in umbra semper, BU ms. 2598, f. $71 \mathrm{v}$.

${ }^{5}$ Sobre este trabajo, cf. Ballesteros 2019.

${ }^{6}$ Acerca de los años parisinos de Casaubon, cf. Ballesteros 2016.

${ }^{7}$ Casaubon 2018, vol. I, p. 61: “[T]he range and sophistication of Casaubon's allusiveness is unusual, and he is at his most allusive with his closest friends. He buries his meaning under layers of erudition, so that only a true soulmate can excavate it. He wants to hide, and he wants simultaneously to be found. For an editor, this is an irresistable prospect".
} 
Antes de presentar el texto de ambas cartas, me gustaría describir brevemente algunos rasgos formales de este trabajo. He intentado respetar los criterios editoriales establecidos por el profesor Solís en el artículo de 1998 donde editó 8 cartas de BU ms. 2598 relacionadas con Justo Lipsio (15471606). Estos criterios consisten en la simplificación de la geminación de la $t$ de littera en la carta de Ramírez de Prado para respetar el uso identificado por Solís de los Santos en este autor, ${ }^{8}$ la regularización de $v / u$ según el uso latino moderno, la sustitución de $j$ por $i$ y el desarrollo de $\&$ como et. No he señalado los signos diacríticos presentes en el manuscrito (tildes, circunflejos) que, a mi juicio, no tienen relevancia en la comprensión del texto latino, ni las tachaduras y enmiendas menores. He agregado las finales $m$, $n$, ue y el enclítico que cuando estaban abreviadas sin señalarlo como abreviatura. Al margen de estas decisiones, he procurado intervenir muy poco en el texto original, cuando lo he hecho ha sido únicamente porque parecía imprescindible: desarrollo de abreviaturas - que pongo entre corchetes [ ] -, restitución de algunas palabras o sílabas perdidas en la transcripción - que señalo entre corchetes angulares $<>-$ y puntuación para entender mi propuesta de lectura. El signo -/- indica el paso de página en el manuscrito. En la sección de comentario se señalan, entre otras cosas, las correcciones propuestas y las intervenciones sobre el original que considero más importantes.

\section{EDICIÓN Y TRADUCCIÓN}

I. [Lorenzo Ramírez de Prado (Salamanca)] a Isaac Casaubon (París) [= nº 58 Solís de los Santos]

[ca. noviembre 1605]

c: Salamanca BU ms. 2598, f. 67r.

Iamdiu in animo habui te (amantissime Casaubone) literis interpellare cum et humanitatem tuam in bonarum literarum amatores cognoverim satis tum quod rationes vitae meae ad te proficisci aliter non patiantur. Sed diu etiam hac cupiditate ardenti commotus collegi ipse me[ri]t[o]s dignitati tuae potius quam desiderio meo 5 decrevi satisfacere. Fregit hoc meum consilium nobilis vir Johannes Bodecker qui tuae comitatis mihi fuit locupletissimus testis. Iam ergo ab eodem via munita aggredior iter ad tuam amicitiam amplectandam et desidero vehementer ut vincula arctiora mutuo nos adstringant. Stipulor conceptissimis verbis o utinam et respondeas! Ego vero pro virili faciam ut ad quaecumque volueris ita sim praesto ut tua

\footnotetext{
${ }^{8}$ Cf. Solís de los Santos 1998, p. 310.
} 
10 caussa omnia non solum debere me quam possem, sed ea quae non possem putem. Cogunt me muti procuratores tui, opera dico quae $\theta \alpha v \mu \alpha \tau o v \varrho \gamma \omega \hat{s}$ de te loquuntur. Summo studio atque industria quaesivi ea omnia et collegi. Nunc Persium et Plinium Secundum a te illustratum video et admiror. Nonne obscuritatis nebula reiecta micat satyrographus radiis ardentissimi solis illustratus! Nos vero parva 15 decent, arbusta iuvant humilesque myricae. En tibi mitto Apologiam in eruditissimi viri $\gamma \varrho \alpha ́ \mu \mu \alpha \tau \alpha$ quam si theta nigro iugulaveris goriae erit Aeneae magni dextra cadam. Sin minus et albo lapillo numeraveris fundam genio merum nec desinam litare tibi, o decus Musarum. Vale.

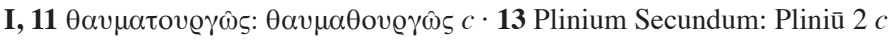

14-15 Nos ... decent.] Hor., ep., 1, 7, 44 (Parvum parva decent) $\cdot 15$ arbusta... myricae.] Verg., ecl., $4.2 \cdot 16$ si ... iugulaveris] Cas. ad Pers., 4, 13 (Et potis es nigrum vitio praefigere theta), p. $327 \cdot \mathbf{1 6 - 1 7}$ Aeneae ... cadam] Verg., aen., 10, $830 \cdot \mathbf{1 7}$ albo ... numeraveris] Cas. ad Pers., 2, 1 (numera meliore lapillo), p. $175 \cdot 17$ fundam ... merum] Cas. ad Pers., 2, 3 (Funde merum Genio), pp. 175-176.

I. [Lorenzo Ramírez de Prado (Salamanca)] a Isaac Casaubon (París) [= nº 58 Solís de los Santos]

[ca. noviembre 1605]

c: Salamanca BU ms. 2598, f. 67r.

Hace tiempo, mi muy amado Casaubon, que tenía intención de dirigirme a ti por carta, tanto por haber conocido tu buena voluntad hacia los amantes de las buenas letras, como por las circunstancias de mi propia vida que irremisiblemente me conducen hacia ti. Pero también es cierto que, impelido por esta ansia ardiente, antes de decidirme a satisfacer mi deseo, desde aquel momento me propuse reunir personalmente merecimientos que se ajustaran a tu dignidad. Ablandó mi determinación el señor don Johannes Bodecker que me proporcionó testimonios inequívocos de tu sencillez. En este momento, en fin, me atrevo a iniciar el camino que él me ha abierto para alcanzar tu amistad y manifiesto vivamente el deseo de que estrechos lazos nos unan a ambos. ¡Me conformo con que, ojalá, me respondas siquiera con unas pocas palabras! Por mi parte, pondré todo el empeño en estar presto a cuanto desees de modo que, con respecto a ti, no solo cuanto esté en mi mano sea para mí una obligación, sino que aquello que no lo esté se convierta en objeto de mis desvelos. A ello me fuerzan tus mudos emisarios, me refiero a tus libros que hablan prodigiosamente de ti. Los he buscado y reunido todos con mucho trabajo y esfuerzo. Ahora leo y admiro el Persio y el Plinio Segundo con tus comentarios. ¡Cómo relumbra el satirógrafo cuando, disipada la bruma de la confusión, es iluminado por los rayos del sol que más brilla! Nosotros, sin embargo, nos conformamos con menos, nos complacen arbustos y humildes tamaris$\cos$. En este sentido te remito una Apología escrita contra cierto eruditísimo caballero, si decides destruirla con la $\theta$ negra, será una bendición caer ante la diestra del gran Eneas. Si, en cambio, la cuentas entre las que merecen la chinita blanca, derramaré vino puro en tu genio y no dejaré de rogar por ti, oh, gloria de las Musas. Adiós. 
II. Isaac Casaubon (París) a Lorenzo Ramírez de Prado (Salamanca) $\left[=n^{\circ} 78\right.$ Solís de los Santos]

31 agosto 1606

c: Salamanca BU ms. 2598, ff. 90v-91r.

Isaacus Casaubonus D[omino] Laurentio Ramirez de Prado, viro nobilissimo s[alutem] $\mathrm{d}[$ icit].

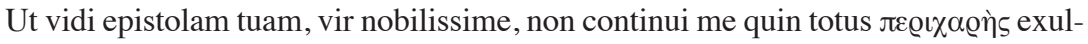
tarem atque adeo insigni ovans gaudio $<$ ut $>$ vix apud me essem. Non enim ab igno5 to mihi viro epistola illa venerat, sed ab eo quem propter singularem et admirabilem eruditionem (nam genus altum pleraque nobilitas censeri postulat praetermitto) diu

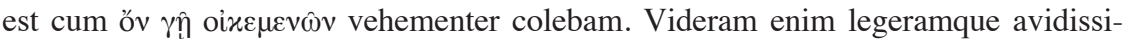
me tuam illam de loco Pliniano diatribam elegantem siquid in literis video et doctam atque ingeniosam. Eius scripti tui lectione ita equidem fueram affectus ut sublatis in

10 caelum manibus omnia tibi fausta ac lata statim precarer, neque ab eo die tuum nomen in ore habere et apud solide doctos celebrare desinerem. O felix vive diu nobilissime iuvenum, et quod c[o]episti facere ad pulcherrimum illud philosophiae et mellitum literarum temperamentum $x \alpha \grave{\text { gú } \gamma \varkappa \varrho \alpha \sigma ı v ~ d u c e m ~ t e ~ p r a e b e ~ H i s p a n i s ~ t u i s ~ q u o r u m ~ u t ~ a c r e ~}$ ingenium, iudicium et subtilitatem in scientiis vulgo omnes laudant. Ita non desunt qui

15 in tam erudito hoc s[a]eculo, linguarum et philologiae notitiam in plerisque v[est]rae gentis scriptoribus desiderent. Quo maior admiratio animum meum incessit cum illam

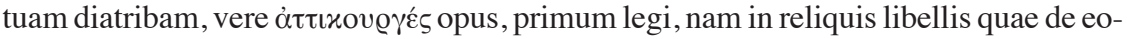
dem argumento scripta hactenus vidi isthi[nc] nuper edita, nihil offendebam quod iure mirarer, nonnulla propter quae iure indignarer. Quis enim recto iudicio qui sit, xaıviेv

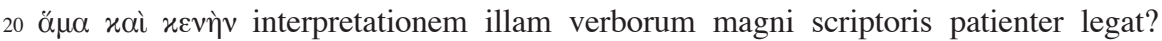
Omitto genus scriptionis cuius profecto gratia nemo paullo doctior vadimonium sit deserturus. Tu et vere sententiam propugnasti et quod paucis clariora latine scribere illum tuum antagonistam docuisti. Denique effecisti tribus chartis ut nihil sit tam arduus in literis quod sperari a te non queat. Id specimen nobilissimi tui ingenii dedisti.

25 Nostras vero scriptiunculas quod tantopere extollis, agnosco benevolentiam tuam. Non negaverim amore ingenti literarum ab ineunti aetate flagrasse me semper, etsi quantum $\mathrm{p}$ [ro]fecerim me sane poeni[tet]-/-. Incidit enim iuventus nostra in tristissima furorum civilium nostrorum tempora ut ad XX aetatis annum prius accederem quam vel de no[min]e bonos auctores haberem notos. Etiam ab illo tempore quibus iactatus

30 fuerim tempestatibus, si dicere aggrediar longum faciam. Itaque non laudem e scriptis nostris exspectamus, sed veniam $\dot{\varepsilon} \varkappa \tau \hat{\eta} \varsigma \hat{\alpha} v \varepsilon \xi$ $\xi x \alpha x i \alpha \varsigma$ et aequanimitate $<$ m $>$ candidorum decorum. Meo q[uo] voto abunde satisfecerit qui egregiae voluntatis in literas et literatos testimonium mihi non inviderit, non cessamus interim ea parare quae non sine fructu aliquo studiosorum $\hat{\omega} \varsigma \dot{\varepsilon} \mu \alpha v \tau o \grave{l} \pi \varepsilon \dot{\imath} \theta \omega$ Deo propitio sumus edituri. Polybium in

35 manibus habemus, scriptorem magnum et parum in vulgus notum. Eum nos vertimus

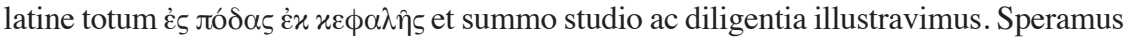
gratum opus fore omnibus qui Musas cum Marte amant coniungere. Veterem enim graecorum et rom[anorum] militiam accurate tractavimus, multa eruimus adhuc incognita, multa castigavimus perperam a viris doctis accepta atque utinam opus editum (quod 
40 tam cito futurum non est) possem ad te mittere. Et faciam profecto animo lubentissimo si viam indicaveris qua tuto curari liber possit. Edituri sumus graece et latine. Praeter quos habemus codices in Regis Christianissimi bibliotheca, alios sumus nati ex Italia ac Germania singulari beneficio duorum Illustrissimorum Principum Bavariae et Urbinatis. Vellem et a te adiuvari possem aliqua re velut si Hygini liber De Castrametatione

45 Ro[manorum $]$ apud vos alicubi lateat, quaeso oroque per omnes Musas exemplum mitte; aut quidquid fuerit eiusmodi amabo commodus imperti. Vale nobilissime iuvenum et me ama, rogatus qui recipisti ultro Lutetiae Parisiorum, a[nte] d[iem] Kal[endas] Sept[embris] MDCVI.

Opus de critica (nam de hoc quaerebas) nondum prodiit. Prodibit si annuerit Deus 50 c[o]eptis mox ab edito Polybio cum libro De coloribus et aliis non paucis quae pridem habemus affectae.

\section{II, 43 Bavariae: Bavarri $c \cdot \mathbf{4 4}$ Castrametatione: Castramelalione $c$}

4 <ut> vix apud me essem: , vix apud me essem c supplevi ex Cas. epist. ad Prideaux, ed. Almeloveen, $n^{\circ}$ 879, p. 528=ed. Botley-Vince 161303 17, p. 478 (Ego vero lecta illa Apologia, ita sum commotus ut vix apud me essem) $\mathbf{1 1}$ apud solide doctos: apud solide declos c correxi ex BU ms. $2598 \mathrm{f}$. $93 \mathrm{r} \cdot \mathbf{1 3}$ mellitum: melipum $c \cdot \mathbf{1 8}$ isthi[ $n c]$ : isthi $c \cdot \mathbf{2 5}$ scriptiunculas: scripiunculas $c \cdot 27$ poeni[tet] supplevi ex Cas. epist. ad Buxtorf, ed Almeloveen, $n^{\circ} 1043, p .606$ (Nam etsi quantum in vestris literis profecerim, me sane poenitet, amavi tamen semper Linguam

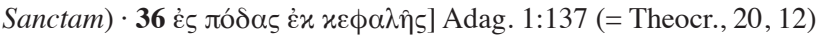

II. Isaac Casaubon (París) a Lorenzo Ramírez de Prado (Salamanca) $\left[=n^{\circ} 78\right.$ Solís de los Santos]

c: Salamanca BU ms. 2598, ff. 90v-91r.

Isaac Casaubon saluda a don Lorenzo Ramírez de Prado, nobilísimo caballero.

Al recibir tu carta, nobilísimo caballero, no pude evitar dar saltos de alegría, llegué incluso a proferir aclamaciones por tan extraordinario gozo hasta el extremo de estar casi fuera de mí. Y es que aquella carta no procedía de un caballero que me fuera desconocido, sino de alguien a quien por su singular y admirable erudición (doy por sentado, desde luego, el hecho de que la mayor parte de la nobleza debe considerarse del estamento superior) yo venía honrando vivamente desde hacía tiempo al ser uno más de los que habitamos esta tierra. Vi, en efecto, y leí con fruición aquella diatriba tuya sobre el pasaje pliniano, ejercicio literario elegante, docto y sutil donde los haya. En efecto, tanto me agradó la lectura de este escrito tuyo que, llevando las manos al cielo, inmediatamente hice votos por tu prosperidad y abundancia. Desde aquel momento, no dejé de tener tu nombre en la boca ni de celebrarlo entre las personas sólidamente doctas. ¡Oh, afortunado, larga vida a ti, el más noble de los jóvenes! Según has emprendido con aquel temperamento y carácter bellísimo para la filosofía y dulce para la literatura, conviértete en el caudillo de tus españoles cuya alabanza 
universal es la de tener aguda inteligencia, buen juicio y sutileza en las ciencias. No faltan en este siglo tan erudito quienes ambicionan el saber filológico y lingüístico propio de la mayoría de los escritores de vuestra nación. Por esta razón se ha hecho mayor la admiración que siento en mi interior en cuanto he leído tu diatriba, trabajo realmente clásico, pues en el resto de tratadillos que he visto escritos hasta ahora sobre el mismo tema y se han publicado por allí, nada encontraba digno de justa admiración, más bien me irritaba alguna que otra cosilla que me iba encontrando. En efecto, ¿habrá alguien dotado de sentido común que lea sin perturbarse una interpretación, tan novedosa como infundada, de las palabras de tan gran autor? Prescindo de las cuestiones de estilo: por respeto al mismo, desde luego, nadie por poco docto que sea debe descuidarse en pulirlo. Tú luchaste por establecer su verdadero significado y a la vez le diste una lección a tu antagonista: en latín con pocas palabras el texto se vuelve más claro. En definitiva, lo resolviste en tres páginas: nada parece tan dificultoso en el mundo de las letras que no pueda esperarse que esté a tu alcance. Tal fue la prueba de tu nobilísimo talento.

Reconozco tu buena voluntad hacia mi persona en el modo exagerado con que ensalzas nuestras notillas. No te negaré que siempre, desde mis primeros años, me ha consumido un gran amor por las letras, si bien lamento lo poco que he aprovechado en este mundo. En efecto, nuestra juventud coincidió con los tiempos funestos de nuestros furores civiles: antes de alcanzar la edad de 20 años no conocía yo a los buenos autores ni de nombre. En fin, si me pongo, mucho podría contar yo de aquel tiempo y de las circunstancias en que me encontré. Por eso no esperamos de nuestros escritos la gloria, sino la tranquilidad que procede de la tolerancia y la serenidad de los elogios sinceros. Con creces cumplirá este deseo mío quien no me mire con malos ojos el testimonio que doy de egregio afecto para con las letras y las personas letradas. En la medida de lo posible, no dejamos de proporcionar pruebas de este tipo que, no sin cierto provecho de los estudiosos, estoy convencido que vamos a editar con el favor de Dios. Tenemos entre manos a Polibio, gran autor y poco conocido entre el público. Estamos traduciéndolo completo al latín, de cabo a rabo, y con gran esfuerzo y atención lo hemos comentado. Confiamos en que el libro sea del gusto de quienes disfrutan con la unión de Marte con las Musas. Hemos tratado con detalle las cuestiones militares de griegos y romanos de la Antigüedad, hemos realizado cantidad de descubrimientos hasta ahora inéditos, hemos depurado cantidad de errores aceptados con descuido por sabios, ojalá, en definitiva, una vez publicado (para lo cual todavía queda un tiempo) pueda yo enviártelo. Con mucho gusto lo haré, desde luego, si me indicas una ruta por la cual el libro pueda transportársete con seguridad. Se tratará de una edición grecolatina. Además de los códices de que disponemos en la biblioteca del Rey Cristianísimo, hemos encontrado otros en Italia y en Alemania gracias al favor excepcional de los Príncipes Ilustrísimos de Baviera y Urbino. Me encantaría que pudieras ayudarme en cierto modo: si, por ejemplo, en algún lugar de tu tierra se ocultara el libro de Higino De castrametatione Romanorum, te pido encarecidamente, por amor de todas las Musas, que me envíes una copia; del mismo modo amaré que me comuniques detalles sobre la existencia de cualquier otra cosa parecida. Adiós, nobilísimo joven, y ámame, te lo ruega quien encontraste lejos de París, a 31 de agosto de 1606.

En cuanto al trabajo de crítica (sobre el que me preguntabas), poco progresa. Si Dios consiente lo que tengo empezado, progresará inmediatamente después de la edi- 
ción de Polibio con el libro De coloribus y con no pocos trabajos que tenemos avanzados desde hace tiempo.

\section{COMENTARIO}

I, 2-3: quod rationes vitae meae ad te proficisci aliter non patiantur.] Lorenzo Ramírez de Prado era el segundo hijo del licenciado Alonso Ramírez de Prado (1549-1608), miembro del Consejo de Hacienda desde 1581 (cf. Entrambasaguas 1943a, p. 19). Su caída en desgracia a partir de diciembre de 1606 con motivo del proceso abierto contra Pedro Franqueza (1547-1614) y contra él mismo por un asunto de malversación y corrupción financiera - “el Desempeño General”, cf. Martínez 2009, pp. 109-111 - será una de las razones por las que su hijo don Lorenzo acabe por renunciar a las vocaciones filológicas y humanísticas que expone a Casaubon. Hasta finales de 1606 lo que sabemos de don Lorenzo tiene que ver exclusivamente con su formación en la Universidad de Salamanca y con su pertenencia a círculos académicos y literarios (cf. Entrambasaguas 1943a, pp. 40-126; Solís de los Santos 2010, 2013 y 2016; Ballesteros 2019). Es muy probable que a partir de este momento don Lorenzo tuviera que dejar a un lado los contactos eruditos que intentó entablar por medio de esta y otras cartas (cf. Solís de los Santos 1998) para centrarse primero en defender la memoria de su padre y, posteriormente, desarrollar una exitosa carrera política.

I, 4: me[ri]t[o]s: MS. mets] Según se ha afirmado en la introducción, el manuscrito BU ms. 2598 es obra de dos escribanos. A diferencia del segundo amanuense, la primera mano, la que ha transcrito esta carta, emplea numerosas abreviaturas. En mi opinión, mets es una de las más complejas. La propuesta "meritos" debe entenderse como una solución en función del sentido de la frase latina.

I, 5-6: nobilis vir Johannes Bodecker qui tuae comitatis mihi fuit locupletissimus testis.] La única información que he encontrado sobre este personaje procede del propio BU ms. 2598. Fue el correo de las misivas a Casaubon (carta 58) y a Lipsio (carta 59) y desde París remitió la carta 80 a Ramírez de Prado, cf. Solís de los Santos 1998, p. 309, n. 106. En la epístola a Lipsio, Ramírez de Prado habla de Bodecker como et nobilis et amici iuvenis [...] cuius ingenitam nobilitatem et orbis perlustrandi cupiditatem nunquam satis laudasse me existimavero (cf. Solís de los Santos 1998, p. 330).

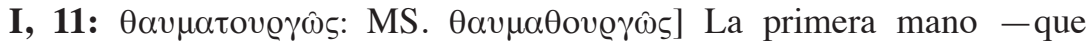
transcribió la epístola de Ramírez de Prado-, a diferencia de la segunda - el transcriptor de la carta de Casaubon - no tiene problemas para leer y transcribir términos en griego, que he normalizado según el uso actual. 
I, 12: Summo studio atque industria quaesivi ea (scil. opera) omnia et collegi.] Lorenzo Ramírez de Prado reunió a lo largo de toda su vida una biblioteca formidable. El Inventario que su viuda ordenó elaborar e imprimir en el contexto de la venta de la misma es fundamental para conocerla. Actualmente, la mayoría de los libros de la colección de Ramírez de Prado se encuentran en la biblioteca de la Universidad de Salamanca (cf. Entrambasaguas 1943b, vol. I, p. xxxiii). En este Inventario se reseñan varias obras de Casaubon: una edición de 1647 de las notas a las Vidas de Suetonio (Entrambasaguas 1943b, vol. II, p. 13), el tratado De satyrica graecorum poesi et romanorum satira libri duo de 1605 (ibid., pp. 29 y 35), la edición comentada a las Sátiras de Persio de 1605 (ibid., p. 35), una de 1612 de la edición anotada a los Deipnosofistas de Ateneo (ibid., p. 74), una edición de 1612 de las notas a Teofrasto (ibid., p. 93), la edición grecolatina de los Discursos de Dion Crisóstomo de Federicus Morellus introducida por una Diatriba de Casaubon de 1604 (ibid., p. 136), la edición de Polibio de 1609 (ibid., p. 166), las notas a Suetonio en una edición plurium commentariis de 1610 (ibid., p. 169), la edición anotada de la Historia Augusta de 1603 (ibid., p. 206) y la edición anotada de las Vidas de Suetonio de 1595 (ibid., p. 207). Sobre el uso que de la edición de la Historia Augusta de Casaubon se hace en los Hypomnemata de 1607, un comentario a los primeros 4 libros de los Epigramas y al De Spectaculis de Marcial publicado por Ramírez de Prado, cf. Ballesteros 2019, §§ 5-8.

I, 12-13: Nunc Persium et Plinium Secundum a te illustratum video et admiror.] Casaubon publicó en 1605 una edición con comentarios de las Sátiras de Persio. Ramírez de Prado cita inmediatamente varios pasajes de estos comentarios casaubonianos. En la reimpresión de 1591 de la edición de las Cartas de Plinio el Joven que hizo su suegro, Henri Estienne ( $c a$. 1531-1598), Casaubon pudo añadir 15 páginas con algunas notas y enmiendas propias (cf. Pattison 1892, p. 476).

I, 15-16: mitto Apologiam in eruditissimi viri $\gamma_{0} \alpha \dot{\mu \mu \alpha \tau \alpha] ~ R a m i ́ r e z ~ d e ~}$ Prado envía a Casaubon una versión de su trabajo en torno a un polémico pasaje del séptimo libro de la Historia Natural de Plinio. Se trata de atque etiam morbus est aliquis per sapientiam mori (Plin., NH, 7, 169) sobre cuya interpretación existe abundante literatura. Además de diversas propuestas de restitución, se ha sugerido la posibilidad de que el texto sea una interpolación (cf. Pigeaud 1993). El fragmento en cuestión recibió particularmente la atención de entornos eruditos y médicos sevillanos desde finales del s. XVI. Es posible que el desarrollo de esta polémica fuera consecuencia de la publicación de un capítulo específico sobre el significado del fragmento pliniano por parte de Jerónimo Mercurial (1530-1606), médico y humanista (cf. Mercurial 1588, p. 129 rv). En Sevilla aparecieron, en efecto, diversos opúsculos y trabajos polémicos que pretendían dar una explicación al pasaje 
(cf. Pérez 2008, p. 992, quien cita obras del jesuita Juan de Pineda, Eduardo Fernández, Francisco Jiménez Guillén y Francisco Duarte y Távora). El trabajo remitido a Casaubon por Ramírez de Prado no puede ser el mismo que se ha conservado en el manuscrito 18630 de la Biblioteca Nacional de Madrid (en adelante BNE). Casaubon, entre otros méritos, elogia la brevedad del texto que ha recibido (II, 23: effecisti tribus chartis), pero el documento de la BNE contiene 21 páginas. El texto de la BNE parece ser la versión definitiva preparada para la imprenta por el destinatario de la misiva de Ramírez de Prado, el doctor Cristóbal Medrano, catedrático de medicina de Salamanca. En cualquier caso, esta versión ofrece algún dato que confirma la información que proporciona la carta 59 a Lipsio sobre la génesis de la investigación de Ramírez de Prado a propósito de Plin., NH, 7, 169. Según esta epístola, la intervención de cierto erudito provocó la reacción de Ramírez de Prado acerca del texto de Plinio. El documento BNE ms. 18630, p. 2, explica de modo similar el inicio de la polémica entre Ramírez de Prado y el círculo sevillano: Iam paene exciderat memoria schediasma quoddam superioribus diebus ad te missum de discussione loci illius pliniani de quo me interpellares, repente mihi data sunt literae hispalensis medici apprime doctis simulque sintagma eruditissimi cuiusdam viri qui exponendi literis iam diu laudem multam, non apud nostrates tantum sed apud exteras nationes reportavit. Et quamvis schediasma meum levi brachio fuerat conscriptum, nec stylo virili compositum, pupugit tamen sapientes hosce viros [...]. En esta polémica se acuñó para Ramírez de Prado un calificativo que le debía acompañar en adelante, BNE ms. 18630, p. 3: Sed doctor ille satis pro imperio reliquos vult sibi credere. Philautiam me laborare dicit, quod eius sententiam non sequar. Verbo pugnat, verbo retundendus est. El hecho de que José Justo Escalígero (1540-1609) vertiera contra el joven Ramírez de Prado el mismo reproche en una carta a Petrus Scriverius (1576-1660) fechada de modo impreciso en 1607 (Praeclarum ingenium est et bona indoles, sed quam depravare possit $\phi \imath \lambda \alpha v \tau i \alpha$, cf. Scaliger 2012, vol. VII, p. 2) demuestra que la filautía ('prepotencia', 'orgullo', 'autosuficiencia', 'narcisismo') de Ramírez de Prado atravesó las fronteras, quizá por mediación del propio Casaubon, cf. Ballesteros 2019, §§ 20-21.

I, 16: si theta nigro iugulaveris] En la Sátira, IV, v. 13 (potis es nigrum vitio praefigere theta, "eres capaz de señalar el vicio con la negra zeta") de Persio en la que se inspira esta expresión, el poeta, por medio de Sócrates, critica a Alcibíades por su dedicación a la política. En su comentario a este verso, Casaubon explica el origen militar de la expresión y el significado de la letra $\theta$ en la misma (es la inicial de zánatos). Casaubon 1605, p. 327: Cum recensebant laterculos militum, nominibus eorum qui perierant, praefigebant $\theta$ : hoc erat expungere. Pero finaliza comentando el valor textual del símbolo $\theta$, que es lo que da sentido a la frase de Ramírez de 
Prado: Sidonius Apolinaris usus est pro nota critica qualis erat graecorum

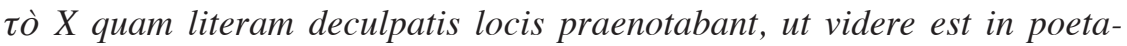
rum scholiis antiquis. A partir de la exégesis casauboniana, Ramírez de Prado construye su frase final y define una intención. Para él, que ha reunido y demuestra haber leído con atención sus libros, será un privilegio el mero hecho de que Casaubon se digne siquiera a considerar prescindible su trabajo anotándolo con el lúgubre signo $\theta$, tal y como suele hacer deculpatis locis. Es significativo del proceso de redacción empleado por Ramírez de Prado y de las intenciones que el mismo oculta que, en la carta 59 a Lipsio, en una expresión prácticamente paralela a la aquí analizada, Ramírez de Prado asocie un verso de Horacio - y no el de Persio- al de Virgilio: Dele, transverso calamo atrum signum allinito: victor abibo cadens Aeneae magni dextra, cf. Solís de los Santos 1998, p. 330 y notas ad hoc.

I, 17: Sin minus et albo lapillo numeraveris fundam genio merum] Esta expresión resulta de la fusión de dos versos de Persio (v. 2, 1: diem numera meliore lapillo, y 2, 3: funde merum genio). De nuevo el comentario de Casaubon, quien relaciona el meliore lapillo de Persio con albo lapillo conocido por otras fuentes (cf. Ruiz de Elvira 2003), puede estar en el origen de la formulación latina empleada por Ramírez de Prado, Casaubon 1605, p.

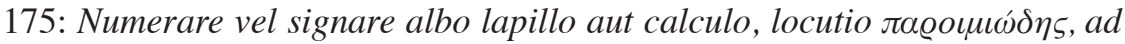
cuiuscunque gaudii significationem. Notissima historia ex Graecis et Plinii septimo. Symmachus duriuscule usurpavit libro primo, epistola xc. En otros textos, Ramírez de Prado vuelve a usar la expresión meliore lapillo/calculo. Por ejemplo, en la carta 62 con la respuesta a Morlanes a la que aludí en la introducción (BU ms. 2598 f. 71v: utinam eundem calculum demereantur quo insignitum gaudet sintagma nostrum coronaque semper redimita exero laetus cultus a culta manu) o en la conclusión del tratadillo sobre Plin., $N H$, 7, 169, conservado en BNE ms. 18630, p. 21: Illud cave existimes me voluisse de summa viri summi eruditione aliquid scriptis meis detractum quem tanti facio ut optaverim eius ingenii luce Academiam nostram esse non destitutam, sed illustratam. Et spero fore qui eius utilitatis publicae amor est ut aliquando ad nos veniat qui dies, si nobis illuxerit, meliore lapillo a me numerabitur. Vale. No obstante, sólo en la carta a Casaubon se utiliza la expresión albo lapillo, y no el meliore lapillo de Pers., 2, 1.

II, 3: non continui me quin] Casaubon usa esta misma expresión para señalar el entusiasmo con que contestó a cierto contacto proporcionado por el helenista augsburguense David Hoeschelius (1556-1617), carta 616, del 30 de noviembre 1608, p. 324, ed. Almeloveen 1709: Non continui me, quin e vestigio ad amplissimum virum literas exararem, testes iudicii mei de illo scripto et Scriptore Thrasonico. 
II, 4: adeo insigni ovans gaudio <ut> vix apud me essem: MS., vix apud me essem.] La desaparición de $u t$ puede haberse debido a la similitud gráfica de $u t$ con el siguiente vi de vix, lo cual habría causado un error por haplografía en la transcripción. Por lo demás considero imprescindible este ut por ser régimen de adeo. Casaubon empleó una estructura muy similar para expresar un momento de perturbación y sorpresa en una carta a John Prideaux (1578-1650), rector del colegio de Exeter (carta 879, p. 528, ed. Almeloveen 1709= carta 161304 17, ed. Botley-Vince 2018, vol. III, p. 478: Ego vero lecta illa Apologia, ita sum commotus ut vix apud me essem, quum reputarem impudentiae huius magnitudinem).

II, 10-11: neque ab eo die tuum nomen in ore habere et apud solide doctos celebrare desinerem: MS. apud solide declos celebrare desinerem] El declos de BU ms. 2598 es claramente una lectura errónea. Casaubon describe su reacción al leer el texto de Ramírez de Prado sobre el pasaje de la Historia Natural de Plinio. Sin duda expresó su aprobación en voz alta. Es posible suplir el texto de la carta de Casaubon gracias a la versión de este momento que dio un testigo del mismo en otra misiva cuya copia se ha conservado en BU ms. 2598. Johannes Bodecker entregó la epístola y el tratado de Ramírez de Prado. Después es muy probable que aguardara a que Casaubon diera lectura a ambos. Él mismo describe ese momento y la reacción de Casaubon, BU ms. 2598, f. 93r: Reddidi quoque Casaubo-

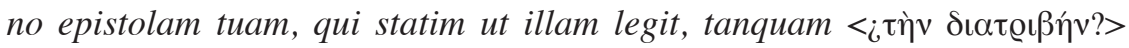
arripuit et multa magni fieri et amici apud solide doctos de te locutus est <vacat? es fin de línea> a nemine expectari possit sancte affirmavi, sed de his et aliis fusius ipse ad te. El copista de esta carta - el segundo amanuense - dejó varios espacios en blanco. Es de suponer que se trata de palabras que no entendía, cf. Solís de los Santos 1998, n. 106. Es factible que algunas de estas palabras ininteligibles estuvieran escritas en griego, por lo que propongo suplir el primer hueco con el término con el que Casaubon definió el opúsculo de Ramírez de Prado, II, 7-8: tuam illam de loco Pliniano diatribam. De un modo quizá inapropiado, Ramírez de Prado, I, 14-15, la había calificado como Apología (tibi mitto Apologiam in eruditissimi viri

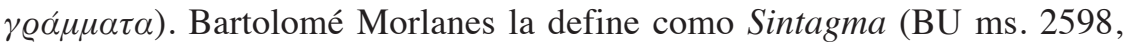
f. 69v: tuum sintagma de loci Pliniani explanatione). El propio Ramírez de Prado llama schediasma a la versión inicial de su trabajo remitida a Medrano (BNE ms. 18630, p. 2: Iam paene exciderat memoria schediasma quoddam superioribus diebus ad te missum de discussione loci illius pliniani de quo me interpellares).

II, 12-13: ad pulcherrimum illud philosophiae et mellitum literarum temperamentum: MS. melipum] Melipum es una lectura incorrecta. Aquí forma parte de la alabanza del temperamento literario de los españoles. Mellitus es un adjetivo que Casaubon emplea en otras ocasiones para referirse a un es- 
tilo terso y elevado como, por ejemplo, el de Sinesio, Casaubon 1600, p. 2: apud mellitissimum Synesium.

II, 12-16: quod c[o]episti facere ad pulcherrimum illud philosophiae et

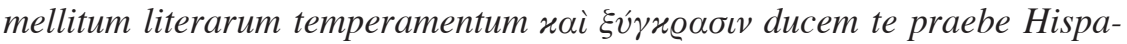
nis tuis quorum ut acre ingenium, iudicium et subtilitatem in scientiis vulgo omnes laudant. Ita non desunt qui in tam erudito hoc s[a]eculo, linguarum et philologiae notitiam in plerisque v[est]rae gentis scriptoribus desiderent.] Entre las cartas que Casaubon recibió desde España, la de Ramírez de Prado es, por ahora, la primera. Luis Tribaldos de Toledo (1558-1634), preceptor del Conde de Villamediana, le escribió desde Madrid el 7 de noviembre de 1606 (cf. carta 569, pp. 300-301, ed. Almeloveen 1709; cf. Tournoy 2002, p. 1257, n. 26. Sobre Tribaldos, cf. Antonio 1996, vol. II, p. 67; Viforcos 2008) y el humanista y canónigo de la catedral de Sevilla Juan Fonseca y Figueroa (1585-1627) le escribió desde Sevilla el 1 de agosto de 1607 (cf. Roe 2012. Sobre Fonseca, Antonio 1996, vol. I, p. 691; Moya 2011). La opinión que aquí expresa Casaubon acerca del carácter de los españoles matiza las afirmaciones en cierto modo contrarias formuladas en la discreta intimidad de su diario personal que, al parecer, circulaban en los cenáculos humanísticos europeos, cf. Casaubon 1850, vol. I, p. 190; Ballesteros 2009 y 2013 , p. 70 , n. 40.

II, 17-19: in reliquis libellis quae de eodem argumento scripta hactenus vidi isthi[nc] nuper edita, nihil offendebam quod iure mirarer, nonnulla propter quae iure indignarer.] Casaubon parece estar informado de la polémica sobre el pasaje de la Historia Natural de Plinio, cf. el comentario a I, 15-16. La errata en la transcripción del isthinc puede deberse al particular ductus casauboniano. En otra carta de Casaubon, su editor encuentra una dificultad similar para leer isthinc, cf. Martin 1890, p. 41.

II, 26-27: etsi quantum p[ro]fecerim me sane poeni[tet]] Para lamentar las carencias de su propia formación, Casaubon usa esta misma expresión en una misiva al hebraísta Johannes Buxtorf (1564-1629), escrita desde París, el 12 de febrero de 1610 (cf. carta 1043, p. 606, ed. Almeloveen 1709: Nam etsi quantum in vestris literis profecerim, me sane poenitet, amavi tamen semper Linguam Sanctam, cf. Grafton-Weinberg 2011, p. 67, n. 25).

II, 27-29: Incidit enim iuventus nostra in tristissima furorum civilium nostrorum tempora ut ad XX aetatis annum prius accederem quam vel de no[min]e bonos auctores haberem notos.] La biografía clásica de Isaac Casaubon sigue siendo la de Pattison 1892. Existen además varios trabajos monográficos sobre diversos momentos de su vida como Tournoy 1998, Canfora 2002, Parenty 2009 o Ballesteros 2016. En su correspondencia, Casaubon ofrece pocos detalles de su propia biografía. En la carta de 1613 a John Prideaux, citada más arriba, se encuentra lo más parecido a un esbozo autobiográfico. Isaac Casaubon nació en Ginebra donde su familia se había 
refugiado por razones religiosas. A los dos años regresó a Francia y a los nueve años ya había aprendido a hablar latín a patre optimo et doctissimo institutus. En ese momento su formación hubo de interrumpirse. Su padre, Arnaldo Casaubon, pastor calvinista, abandonó su hogar en 1568 para participar en las Guerras de Religión durante tres años. Las masacres sufridas por los calvinistas a partir de la noche de San Bartolomé en 1572 de nuevo obligaron a la familia Casaubon a huir, esta vez hacia el sur de Francia. Arnaldo Casaubon aprovechó la huida para enseñar a su hijo los rudimentos del griego. En 1578 Isaac fue enviado a estudiar a Ginebra donde 5 años más tarde se convirtió en profesor de griego. Durante los 14 años siguientes (1583-1596), desarrolló su carrera académica en ese lugar. Allí recibió la protección y las enseñanzas del teólogo Teodoro de Beza (1519-1605) y de Julio Pace (1550-1635), traductor y comentarista de Aristóteles. Entre 1596 y 1599, Isaac Casaubon se instaló en Montpellier. En enero de 1599 recibió la invitación de Enrique IV para trasladarse a París, donde vivió la década posterior. En 1610, después del asesinato del rey, recibió permiso para viajar a la corte de Jacobo I de Inglaterra. Allí vivió los últimos años de su vida (cf. ed. Botley-Vince 2018, vol. III, pp. 472-479; Tournoy 1998, pp. 192-193).

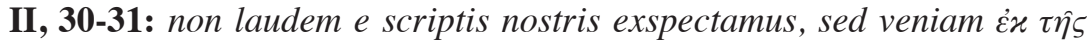

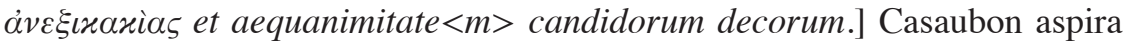
a que su obra le proporcione tolerancia y tranquilidad. Ramírez de Prado no debía desconocer las complejas circunstancias religiosas en las que se desarrollaba la vida parisina de Casaubon. Calvinista al servicio de un rey católico, Casaubon sobrevivió a las tensiones que su condición le generó instalándose en un exilio interior frente a papistas y protestantes, y aceptando su situación como un martirio (cf. Ballesteros 2016, pp. 89-104 y 121-126). En una carta al humanista protestante Georg Michael Lingelsheim (1556-1636), fechada en París, el 30 de marzo de 1606, Casaubon ya utilizó el término $\alpha \dot{v} \varepsilon \xi เ x \alpha x i ́ \alpha$ para describir la misma necesidad de respeto y tolerancia (carta 495, p. 263, ed. Almeloveen 1709: Ego spem omnem meam in

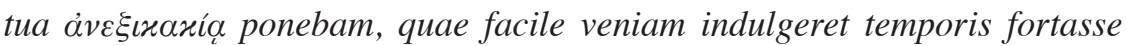
non optime positi). En los repertorios lexicográficos contemporáneos, la $\alpha \dot{v \varepsilon} \xi \iota x \alpha x i ́ \alpha$ es la virtud equivalente a la tolerancia, la clemencia o la resignación. El Thesaurus de Henri Estienne la define como "Tolerantia, Patientia malorum, iniuriarum" (Estienne 1835, vol. 1.2, cols. 696-697), el de Buenaventura Vulcanius (1538-1614) como "Clemencia, Paciencia" (Vulcanius 1600, col. 375). Es posible que, tal y como se emplea en la carta a Ramírez de Prado - en la que las aspiraciones de Casaubon se expresan por

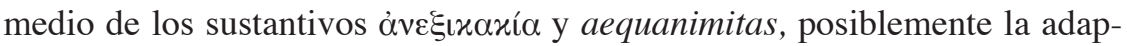
tación casauboniana del término $\mu \alpha x \varrho o \theta v \mu i ́ \alpha-$, nos encontremos ante una alusión a un texto del Contra Celso de Orígenes (2, 34, 40-41, ed. Borret 


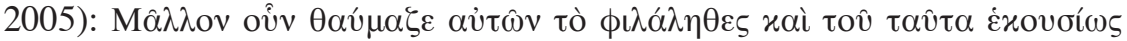

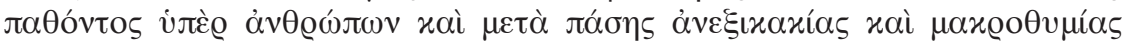

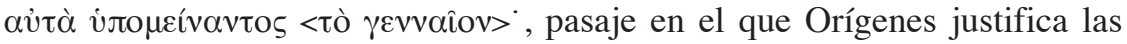
conductas dignas de Dios: "Admira pues la sinceridad de estos autores y la excelencia de quien ha aceptado voluntariamente los sufrimientos de los hombres y los ha soportado con resignación y grandeza de espíritu" (trad. nuestra). Casaubon conocía bien la edición de Hoeschelius del Contra Celso (1605) de cuyas pruebas acusa recibo en una carta al propio Hoeschelius fechada en París, el 18 de febrero de 1604 (carta 384, p. 204, ed. Almeloveen 1709: Accepi hodie utrumque simul fasciculum tuum. Gaudeo impositum a te finem libris eruditissimis contra Celsum quorum lectione mirum in modum dum delectatus ut prioribus meis significavi; carta 385, p. 204, del 21 de febrero de 1604, ed. Almeloveen 1709: Incredibilem in modum delectarunt me tum Origeniaca quae nondum legeram...). En la edición de Hoeschelius al texto griego se añadió la traducción latina de Segismundo Gelenio (ca. 1498-1554) que para el pasaje referido es: Mirare potius eorum veracitatem, ipsumque, qui sponte haec passus est pro hominibus summaque longanimitate ac tolerantia pertulit (Origenes 1605, p. 83).

II, 34-35: Polybium in manibus habemus] La edición y traducción latina de las Historias de Polibio es la última obra publicada por Casaubon durante su período parisino. Al menos desde 1595 tenía la intención de publicar la obra de Polibio (Tournoy 1998, p. 201), pero su edición sólo apareció en la imprenta de Jerónimo Drouart en 1609. Su edición incluye por primera vez los cinco primeros libros de las Historias, conservados completos, los extractos de los libros VI a XVIII conocidos como Excerpta antiqua, los Excerpta legationum y los fragmentos polibianos transmitidos por tradición indirecta. Este trabajo, que constituye uno de los grandes monumentos de la filología humanística, inauguró la crítica de Polibio (cf. Polybe 2003, pp. lcv-lcvi).

II, 39: multa castigavimus perperam a viris doctis accepta] La edición príncipe del texto griego de Polibio (Haguenau, 1530) fue obra de Vicente Opsopoeus $(\dagger 1539)$. En su edición de Basilea (1549), Johannes Hervagius (1497-1558) publicó por primera vez los Excerpta antiqua (Tournoy 1998, p. 198). Ambas se consideran prescindibles por sus errores e incorrecciones (cf. Polybe 2003, p. lxv). En una carta a Jacques Bongars (1546-1612), alumno de Cuias y embajador de Enrique IV, Casaubon se mostró también crítico con el De militia romana de Lipsio, una traducción comentada de los capítulos 19 a 40 del libro VI de las Historias de Polibio publicada en 1595 (carta 21 del 15 de diciembre de 1595, p. 15, ed. Almeloveen 1709: cur hanc potissimam rationem tractandi eius argumenti elegerit? [...] Mitto errores aliquot mihi notatos in explicandis locis graecorum scriptorum, neque nos tamen studiose quaesivimus, sed casu in illos incidimus, cf. Martin 1890, pp. 3-4, y Tournoy 1998, p. 200). 
II, 41-44: Praeter quos habemus codices in Regis Christianissimi bibliotheca, alios sumus nati ex Italia ac Germania singulari beneficio duorum Illustrissimorum Principum Bavariae et Urbinatis.] Casaubon alude a alguno de los manuscritos usados en su edición. En la nota "De codicibus mss. quorum in recensendo Polybio fuit nobis copia" que precede a su edición, Casaubon describió los códices que empleó en las tres partes de su trabajo (cf. Polybius 1609, s. p.). Aquí refiere Ramírez de Prado a manuscritos colacionados en las dos primeras partes: la edición de los cinco primeros libros completos de las Historias y la de los Excerpta sive epitomata (la tercera parte la componen los excerpta legationum y los fragmenta e variis scriptoribus collecta). En la primera parte, Casaubon recurrió a un libro Regiae Bibl[iothecae] elegantissime descripto, sed manus recentissimae cui parilem dono dedit nobis olim vir nobilis Antonius Verderius Lugdunensis, sed in quo tres postremi libri deerant. His duobus vetustior est saeculo uno vel altero quem nacti sumus e bibliotheca Serenissimi Principis Bavariae Ducis. El manuscrito regio manus recentissimae - del que procedía la copia incompleta donada por Antoine du Verdier (1544-1600) - puede ser el Parisinus gr. 1649 (Regius B) de 1547 (cf. Polibio 2008, p. lxxxv), uno de los siete manuscritos polibianos parisinos conocidos que contienen los cinco primeros libros de las Historias (cf. Moore 1965, p. 184). El Parisinus gr. 1648 (Regius A), por ejemplo, es más antiguo - se fecha a fines del s. XIV o principios del xv (cf. Moore 1965, p. 184; Polibio 2008, p. lxxiii)-. El manuscrito quem nacti sumus e biblioteca Serenissimi Principis Bavariae Ducis o codex Bavaricum, según lo denomina en una carta a José Escalígero (carta 510, p. 269, ed. Almeloveen 1709= 160607 06, ed. Botley-Van Miert 2012, vol. VI: Exspecto nunc codicem Bavaricum, si tantum apud Marcum Velserum gratia possum ut eius opera impetrare illum queam), lo consiguió Casaubon través del erudito augsburguense Marcus Welser (1558-1614) de la biblioteca de los Duques de Baviera creada a partir de la del duque Alberto V (carta enviada por Welser a Escalígero 160605 31, ed. Botley-Van Miert 2012, vol. VI: Casaubono me Polybium membranaceum ex Boica bibliotheca impetraturum prope confido). Con toda probabilidad este códice es el Monacensis gr. 157 que también había servido para realizar la princeps de los 5 primeros libros de las Historias polibianas (Polibio 1972, p. cxlvii, y Polibio 2008, p. 1xxii). Para editar los Excerpta antiqua, Casaubon empleó, entre otros, libro m[anu]s[cripto] regiae Bibliotheca non admodum bonae notae. Item vetustissimis membranis Epitomen continentibus librorum XVII primorum e bibliotheca Serenissimi Principis Urbini Ducis. En la Biblioteca Nacional de París se conservan 12 ms. con los Excerpta Antiqua (cf. Moore 1965, pp. 185-186). Desconozco cuál de ellos es este ms. non admodum bonae notae. El Polibio urbinate es más fácil de identificar. Fue prestado a Casaubon por el Duque de Urbino, Francesco María II, gracias a 
la mediación del embajador francés en Venecia Philippe Canaye de Fresne (1551-1610), durante mucho tiempo amigo de Casaubon, y a la del propio rey Enrique IV. Casaubon pudo trabajar con él desde junio de 1606. Fue devuelto en septiembre de 1609 (cf. Martin 1890, pp. 20 y 24). Actualmente se encuentra en la Biblioteca Vaticana. Se trata del "muy valioso" Urbinas gr. 102 (cf. Polibio 1972, p. cxliv; Polybe 2003, p. liii, y Polibio 2008, p. lxxiii).

II, 44-46: si Hygini liber De castrametatione Ro[manorum] apud vos alicubi lateat, quaeso oroque per omnes Musas exemplum mitte] Entre las tres obras atribuidas a Higino Gromático, conservadas en el Corpus Agrimensorum, existe un tratado sobre las técnicas romanas de castramentación: el De munitionibus castrorum (cf. Hyginus 1848, pp. iii-viii y 1-63). En el momento en que Casaubon escribía a Ramírez de Prado no existía una edición impresa de éste por lo demás muy complejo tratado, lo habitual era manejar copias manuscritas del mismo. Es así como lo utilizó Lipsio que lo cita frecuentemente en De militia romana de 1595, su comentario a Polibio (Lipsius 1675, p. 250= De mil., 5, 3: Nisi quis alius in verbis iis [Hyg. De munitionibus castrorum, 18] sensus, quem scrutari magis pensiculate haud possim quoniam scriptus ille codex non ultra mihi ad manum, et paucula sunt excerpta. Siquis habet, et tanti est, videto, imo edito et videndum etiam aliis praebeto). Sin duda Casaubon, al igual que había hecho Lipsio, deseaba utilizar esta obra para el comentario a Polibio con el que proyectaba rematar su edición y traducción de 1609, comentario que nunca completó y del que sólo póstumamente se publicaron las notas al libro I. Muy poco después apareció la edición príncipe del De munitionibus castrorum de Higino, obra de Petrus Scriverius (1576-1660), quien lo publicó en 1607 junto a otros autores romanos de temas militares. Casaubon agradecía el envío de un ejemplar al propio Scriverius por medio de una carta fechada en París el 18 de octubre de 1607 (carta 575, p. 304, ed. Admeloveen 1709: Multum tibi debeo, doctissime Scriveri, propter illud munus eximium quo sum nuper

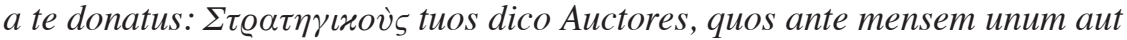
alterum accepi tuo nomine a clarissimo Baudio mihi missos).

II, 46-48: Vale nobilissime iuvenum et me ama, rogatus qui recipisti ultro Lutetiae Parisiorum, a[nte] d[iem] Kal[endas] Sept[embris] MDCVI.] El escribano que copió esta carta no entendió bien la expresión rogatus qui recipisti ultro Lutetiae Parisiorum. Inicialmente dejó un hueco y no escribió las palabras qui recipisti. Posteriormente, con otra tinta, el hueco se completó posiblemente por una mano distinta. El Lutetia' de la transcripción está subrayado. Desde finales de agosto de 1606, Isaac Casaubon se instaló junto a su familia en La Bretonnière, cerca de Chartres al suroeste de París, huyendo de un brote de peste que afectaba a la capital (carta 517 a Escalígero, del 26 de agosto de 1606, p. 272, ed. Almeloveen 1709=1606 
08 26, ed. Botley-Van Miert 2012, vol. VI: Coeterum moestissimus haec tibi scribo, sub discessum in proximum pagum, Bretoneriam nomine). Según la datación de su correspondencia, la familia Casaubon regresó a París a finales de octubre del mismo año. La última carta de Casaubon fechada en La Bretonnière es del 29 de octubre de 1606 (cf. carta 534 a Charles Labbé, p. 279, ed. Almeloveen 1709).

II, 50: libro De coloribus] Según se deduce de una nota de sus Animadversiones a Ath., Deipn., 1, 26A ("[El vino] pone la sangre roja y fluida, y procura sueños tranquilos", trad. de Rodríguez-Noriega), Casaubon tenía ya preparado un comentario a este tratado de Teofrasto, trabajo que, según esta posdata a Ramírez de Prado, Casaubon prevé retomar al finalizar su Polibio, Casaubon 1600, p. 37= 1, 20: In Theophrasti libello de coloribus

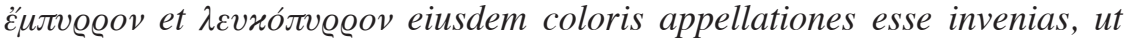
scripsimus in nostro de coloribus commentario. Casaubon había traducido y comentado los Caracteres de Teofrastro en 1592 (Pattison 1892, p. 477).

\section{BIBLIOGRAFÍA}

\section{Fuentes antiguas}

Ateneo, Banquete de los eruditos. Libros I-II, intr., trad. y notas Lucía RodríguezNoriega Guillén, Madrid, Gredos, 1998.

Auli Persi Flacci Satirarum liber, ed. et com. Isaac Casaubon, Parisiis, apud Ambrosium et Hieronymum Drouart, 1605.

Horace, Satires. Epistles. Ars Poetica, ed. George P. Goold, transl. Henry Rushton Fairclough, Cambridge-London, Harvard University Press, 1999 ( $1^{\text {a }}$ ed. 1926, LCL).

Hyginus Gromaticus, Liber De munitionibus castrorum, ed. Christian Conrad Ludwig Lange, Göttingen, apud Vandenhoeck at Ruprecht, 1848.

OrigÈne, Contre Celse. Tome I (livres I et II), intr., texte critique, trad. et notes Marcel Borret, Paris, Sources Chrétiennes, 2005 (1967).

Origenes, Contra Celsum libri VIII, ed. David Hoeschelius, trad. lat. Sigismund Gelenius, Augsburg, ad insigne pinus, 1605.

Pline L'Ancien, Histoire Naturelle. Livre VII, ed., trad. fr. et com. Robert Schilling, Les Belles Lettres, 2003.

Polibio, Historias. Libro I (caps. 1-31), intr., ed. y trad. cast. Alberto Díaz Tejera, Madrid-Barcelona, Consejo Superior de Investigaciones Científicas (Alma Mater), 1972.

Polibio, Historias. Libros V-VI, intr., ed. y trad. cast. Antonio Sancho Royo, Madrid, Consejo Superior de Investigaciones Científicas-Tirant Lo Blanch, 2008.

Polybe, Histoires. Introduction générale. Livre I, ed. et trad. Paul Pédech, Paris, Les Belles Lettres, 2003. 
Polybir Historiarum libri qui supersunt, ed. y trad. lat. Isaac Casaubon, Paris, apud Hieronymum Drouardum, 1609.

Theocritus, The Greek Bucolic Poets, ed. George P. Goold, transl. John Maxwell Edmonds, Cambridge-London, Harvard University Press, 1996 (1912, LCL).

Virgile, Bucoliques, ed. et trad. Eugène de Saint-Denis, Paris, Les Belles Lettres, 2005 (1942).

Virgilio, Eneida IV. Libros X-XII, ed., trad. cast. y not. Luis Rivero García, Juan A. Estévez Sola, Miryam Librán Moreno y Antonio Ramírez de Verger, Madrid, Consejo de Investigaciones Científicas, 2011.

\section{Fuentes modernas}

Antonio, Nicolás, Bibliotheca Hispana Nova, 2 vols., Madrid, Visor, 1996 (facs. orig. 1783).

Ballesteros, Juan Ramón, “ 'Ignorans et barbares': los españoles vistos por Escalígero y otros prejuicios perdurables", Los Papeles Mojados de Río Seco, 8, 2009, pp. 27-29.

Ballesteros, Juan Ramón, "Bárbaros elocuentes y salvajes silenciosos en la Antigüedad y en el Humanismo", Estudios Clásicos, 144, 2013, pp. 57-79.

Ballesteros, Juan Ramón, “Casaubon en París: La anotación de la Historia Augusta (1603) de Isaac Casaubon en la polémica religiosa de tiempos de Enrique IV de Francia", Quaderni di Storia, 84, 2016, pp. 83-126.

Ballesteros, Juan Ramón, "Facete et tecte: Exploraciones barrocas por la poesía de Marcial en los Hypomnemata (1607) de Ramírez de Prado", e-Spania. Revue Interdisciplinaire d'Études Hispaniques Médiévales et Modernes, 32, 2019, $\S \S 1-35$, https://journals .openedition .org/e-spania/29630?lang=en (25/08/19).

CAnfora, Luciano, Convertire Casaubon, Milano, Adelphi, 2002.

Casaubon, Isaac, Animadversionum in Athenaei Dipnosophistas libri XV, Lyon, apud Antonium de Harsy, 1600.

CaSAUBon, Isaac, Epistolae, insertis ad easdem responsionibus quotquot hactenus reperiri potuerunt secundum seriem temporis accurate digestae, ed. Theodoor Jansson Van Almeloveen, Roterodami, Typis Casparis Fritsch et Michaelis Böhm, 1709.

Casaubon, Isaac, Ephemerides, 2 vols., ed. and notes Johanne Russell, Oxford, E Typographeo Academico, 1850.

Casaubon, Isaac, The Correspondence of Isaac Casaubon in England, 4 vols., ed. Paul Botley, Máté Vince, Genève, Droz, 2018.

Entrambasaguas, Joaquín de, Una familia de ingenios. Los Ramírez de Prado, Madrid, Consejo Superior de Investigaciones Científicas-Patronato Menéndez y Pelayo-Instituto Antonio Nebrija-Revista de Filología Española, Anejo 26, 1943a.

Entrambasaguas, Joaquín de, La biblioteca de Ramírez de Prado, 2 vols., Madrid, Consejo Superior de Investigaciones Científicas-Instituto Nicolás Antonio, 1943b. 
Estienne, Henri, Thesaurus Graecae Linguae, 9 vols., ed. Charles Benedict Hase, Wilhelm Dindorf, Ludwig August Dindorf, Paris, Ambrosius Firmin Didot, 1835.

Grafton, Anthony, and Joanna WeInBerg, "I have always loved the Holy Tongue". Isaac Casaubon, the Jews, and a Forgotten Chapter in Renaissance Scholarship, Cambridge-London, Belknap, 2011.

Lipsius, Iustus, De militia romana libri quinque. Commentarius ad Polybium, en Opera omnia, vol. III.1, Wesel, 1675 (facs. Hildesheim, 2003) (1595).

Martin, Albert, "L'édition de Polybe d'Isaac Casaubon (1594-1609)", Mélanges d'archéologie et d'histoire, 10, 1890, pp. 3-43, DOI: 10.3406/mefr.1890.6626.

Martínez Hernández, Santiago, Rodrigo Calderón. La sombra del valido. Privanza, favor y corrupción en la corte de Felipe III, Madrid, Marcial Pons, 2009.

Mercurialis, Hieronymus, Variarum lectionum in medicinae scriptoribus et aliis, libri sex, Venetia, 1588.

Moore, John M., The Manuscript Tradition of Polybius, Cambridge, Cambridge University Press, 1965.

Moya, Francisca, "Fonseca y Figueroa y el texto de las Tragedias de Séneca", en Jesús de la Villa Polo, José Francisco González Castro y Gregorio Hinojo Andrés (coords.), Perfiles de Grecia y Roma. Actas del XII Congreso Español de Estudios Clásicos, vol. III, Madrid, Sociedad Española de Estudios Clásicos, 2011, pp. 285-293.

Parenty, Hélène, Isaac Casaubon helléniste. Des studia humanitatis à la philologie, Genève, Droz, 2009.

Pattison, Mark, Isaac Casaubon, 1559-1614, Oxford, Clarendon Press, 1892 ( $2^{\mathrm{a}}$ ed.).

Pérez Custodio, Violeta, "Plinio el Viejo y los progymnasmata: la edición complutense de la Naturalis Historia de 1569", en José María Maestre Maestre, Joaquín Pascual Barea y Luis Charlo Brea (coords.), Humanismo y pervivencia del mundo clásico. Homenaje al profesor Antonio Prieto, vol. IV.2, AlcañizMadrid, Instituto de Estudios Humanísticos-Consejo Superior de Investigaciones Científicas, 2008, pp. 973-996.

Pigeaud, Jackie, "Un locus desperatus chez Pline l'Ancien", Helmantica, 54, 1993, pp. 467-476.

Ramírez de Prado, Lorenzo, Carta latina al D[octo]r Cristóbal de Medrano sobre el pasaje de Plinio "Atque etiam morbus es $<t>$ aliquis per sapientiam mori", Biblioteca Nacional de Madrid, ms. 18630.

RoE, Jeremy, "New Documents on the Scholarship of Juan de Fonseca y Figueroa (1585-1627): Arabist and Correspondent with Isaac Casaubon", Humanistica Lovaniensia. Journal of Neo-Latin Studies, 61, 2012, pp. 371-388.

Ruiz De Elvira, Antonio, "Albo lapillo", Cuadernos de Filología Clásica. Estudios Latinos, 23/1, 2003, pp. 37-43, https://revistas.ucm.es/index.php/CFCL/article/ view/CFCL0303230037A (25/09/2019).

SCAliger, Joseph Justus, The Correspondence of Joseph Justus Scaliger, 8 vols., ed. Paul Botley and Dirk Van Miert, Genève, Droz, 2012.

Solís de los Santos, José, "Dos cartas desconocidas de Justo Lipsio y otras seis que le atañen en la correspondencia de Lorenzo Ramírez de Prado (1583-1658)", Humanistica Lovaniensia, 47, 1998, pp. 278-331. 
Solís de los Santos, José, "Ramírez de Prado, Lorenzo (1583-1658)", en Diccionario Biográfico Español, Madrid, Real Academia de la Historia, 2010, dbe.rah. es $(25 / 08 / 2018)$.

Solís de los Santos, José, "Ramírez de Prado, Lorenzo (1583-1658)", en José Francisco Domínguez Domínguez (ed.), Diccionario biográfico y bibliográfico del Humanismo español (siglos XV-XVII), Madrid, Ediciones Clásicas, 2013, pp. 698-703.

Solís de los Santos, José, "Cervantes y el entorno humanista de los Ramírez de Prado", Edad de Oro, 35, 2016, pp. 97-120, DOI: 10.15366/edadoro2016.35.005.

TouRnOY, Gilbert, “ 'Ad ultimas inscitiae lineas imus'. Justus Lipsius and Isaac Casaubon in the Changing World of Classical Scholarship", in Marc Laureys (ed.), The world of Justus Lipsius: A contribution towards his intellectual biography. Proceedings of a colloquium held under the auspices of the Belgian Historical Institute in Rome (Rome, 22-24 May 1997), Brussels-Rome, Institut Historique Belge de Rome, 1998.

Tournoy, Gilbert, "A Correspondent of Lorenzo Ramírez de Prado and of Justus Lipsius: Robert de Scheilder”, en José María Maestre Maestre, Joaquín Pascual Barea y Luis Charlo Brea (coords.), Humanismo y pervivencia del mundo clásico. Homenaje al profesor Antonio Fontán, vol. III.3, Alcañiz-Madrid, Instituto de Estudios Humanísticos-Laberinto-Consejo Superior de Investigaciones Científicas, 2002, pp. 1249-1261.

VAN Miert, Dirk, "José Justo Escalígero (1540-1609): Los contactos católicos de un erudito calvinista", en Juan Ballesteros y Juan Manuel Cortés Copete (eds.), Letras peligrosas: Humanistas, religión romana y cristianismo primitivo (= Revista de Historiografía, 11, 2009), Madrid, Actas Editorial, 2009, pp. 87-93.

Viforcos Marinas, María Isabel, "Reflexiones en torno al cronista Luis Tribaldos de Toledo y su Historia de Chile", en Jesús María Nieto Ibáñez y Raul Manchón Gómez (eds.), El Humanismo español entre el Viejo Mundo y el Nuevo, LeónJaén, Universidad de León, 2008, pp. 491-502.

Vulcanius, Bonaventura (ed. et not), Thesaurus utriusque linguae, hoc est Philoxeni, aliorumque veterum authorum glossaria latino graeca et graeco latina. Isidori Glossae latinae; Veteres Grammatici latini et graeci qui de proprietate et differentiis vocabulorum vtriusque linguae scripserunt, Lugduni batavorum, excudebat Ioannis Patius, 1600.

$$
* * *
$$

JuAn RAMÓn BALLESTERos SÁNChEZ es doctor por la Universidad de Huelva (mención doctorado europeo, especialidad Historia Antigua). Es profesor asociado del Área de Historia Antigua de la Universidad Pablo de Olavide (Sevilla). Sus principales líneas de investigación son la Historiografía antigua, la Tradición clásica y el Humanismo europeo. Entre sus publicaciones recientes se encuentran: "Lipsio y las fuentes bizantinas: una nota sobre algunas lecturas de don Baltasar de Zúñiga", $\mathrm{Hu}$ manistica Lovaniensia, 64, 2015, pp. 223-232, "Casaubon en París: La anotación de la Historia Augusta (1603) de Isaac Casaubon en la polémica religiosa de tiempos 
de Enrique IV de Francia", Quaderni di Storia, 84, 2016, pp. 83-126, y "Facete et tecte: Exploraciones barrocas por la poesía de Marcial en los Hypomnemata (1607) de Ramírez de Prado", e-Spania. Revue Interdisciplinaire d'Études Hispaniques Médiévales et Modernes, 32, 2019, §§ 1-35. 